\title{
Optical and X-ray observations of the two BL Lac objects OJ 287 and MS 1458+22
}

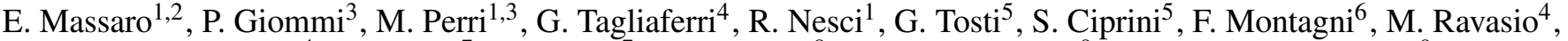 \\ G. Ghisellini $^{4}$, A. Frasca ${ }^{7}$, E. Marilli ${ }^{7}$, G. Valentini ${ }^{8}$, O. M. Kurtanidze ${ }^{9}$, and M. G. Nikolashvilii ${ }^{9}$ \\ 1 Dipartimento di Fisica, Università "La Sapienza”, Piazzale A. Moro 2, 00185 Roma, Italy \\ 2 Istituto di Astrofisica Spaziale, CNR, Via del Fosso del Cavaliere 100, 00113 Roma, Italy \\ 3 ASDC, Agenzia Spaziale Italiana, Frascati, Italy \\ ${ }^{4}$ Osservatorio Astrofisico di Brera, via E. Bianchi 46, 23807 Merate, Italy \\ 5 Dipartimento di Fisica e Osservatorio Astronomico, Universitá di Perugia, via A. Pascoli, 06100 Perugia, Italy \\ ${ }^{6}$ Stazione Astronomica di Vallinfreda, Italy \\ 7 Osservatorio Astrofisico di Catania, via S. Sofia 78, 95123 Catania, Italy \\ 8 Osservatorio Astronomico di Collurania, Teramo, Italy \\ 9 Abastumani Astronomical Observatory, 383762 Abastumani, Georgia
}

Received 23 July 2002 / Accepted 30 October 2002

\begin{abstract}
We present the results of recent BeppoSAX observations of the two BL Lac objects OJ 287 and MS $1458+22$ in bright optical states and of simultaneous photometric measurements in the bandpasses from $I$ to $U$. OJ 287 was observed in November 2001 and MS $1458+22$ in February of the same year. The X-ray flux of OJ 287 was rather low, $F(2-10 \mathrm{keV})=$ $(1.35 \pm 0.15) \times 10^{-12} \mathrm{erg} \mathrm{cm}^{-2} \mathrm{~s}^{-1}$ and its spectrum is well described by a single power law with an energy index $0.45 \pm 0.08$, flatter than that measured in the optical, equal to $1.53 \pm 0.03$. The X-ray emission of MS $1458+22$ was characterized by a low flux level of $F(2-10 \mathrm{keV})=(7.5 \pm 0.6) \times 10^{-13} \mathrm{erg} \mathrm{cm}^{-2} \mathrm{~s}^{-1}$, with a steeper spectral index of $1.71 \pm 0.15$, while in the optical it was $0.99 \pm 0.06$. We show that the broad-band SEDs of these two sources can be well represented by log-parabolic spectral laws and evaluate their parameters. This law, already observed for the synchrotron components of other BL Lac sources, can be explained if the emitting particles are accelerated by some statistical mechanism having a probability of energy gain that is a decreasing function of the energy itself.
\end{abstract}

Key words. galaxies: active - galaxies: individual: OJ 287, MS 1458+22 - techniques: photometry - X-rays: galaxies

\section{Introduction}

Simultaneous multiband observations of BL Lac objects are useful for the understanding of the physical processes responsible for their highly variable emission. In particular, for the so-called LBL (Low frequency peaked BL Lac, Padovani \& Giommi 1995) sources the X-ray band is important because in this energy range it is occasionally possible to detect at the same time both the Synchrotron and inverse Compton (hereafter IC) components, as in the case of ON 231 (Tagliaferri et al. 2000). Previous observations have shown that the former component is generally more variable than the latter and its intensity is correlated with the optical brightness, while the origin of the variations of the IC component remains not well established. Particularly, it is not clear which are the seed photons upscattered into the X-ray range (see for instance Giommi et al. 1999). The study of the variability of X-ray emission, possibly on different time scales, is therefore useful to gain more information about flux correlation between the various

Send offprint requests to: E. Massaro,

e-mail: massaro@astro.uniroma1.it spectral bands. To this end, we performed a few TOO (Target of Opportunity) BeppoSAX pointings of some bright BL Lac objects, whose behaviour in the radio and optical bands has been well known for several years because they are often monitored. In this paper we present the results of the observations of the sources OJ 287 and MS 1458+22, which were observed in bright optical states and showed quite different properties in the $\mathrm{X}$-ray range.

The BL Lac object OJ 287 ( $z=0.306$; Stickel et al. 1989) is one of the best studied sources of this class and, in particular, it is the main target of an intensive optical (Pursimo et al. 2000) and radio (Valtaojoa et al. 2000) monitorings, the former carried out by a large international collaboration. One of its most relevant characteristics is the periodicity of 11.95 years, detected from the historic optical light curve (Sillanpää et al. 1988). A light curve in the $R$ (Cousins) band, spanning the time interval from 1993, one year before the large maximum, to 2001 is shown in Fig. 1.

X-ray observations of OJ 287 were performed on several occasions with different satellites, starting from the first detection with Einstein in 1979-1980 (Madejski \& Schwartz 1988). 


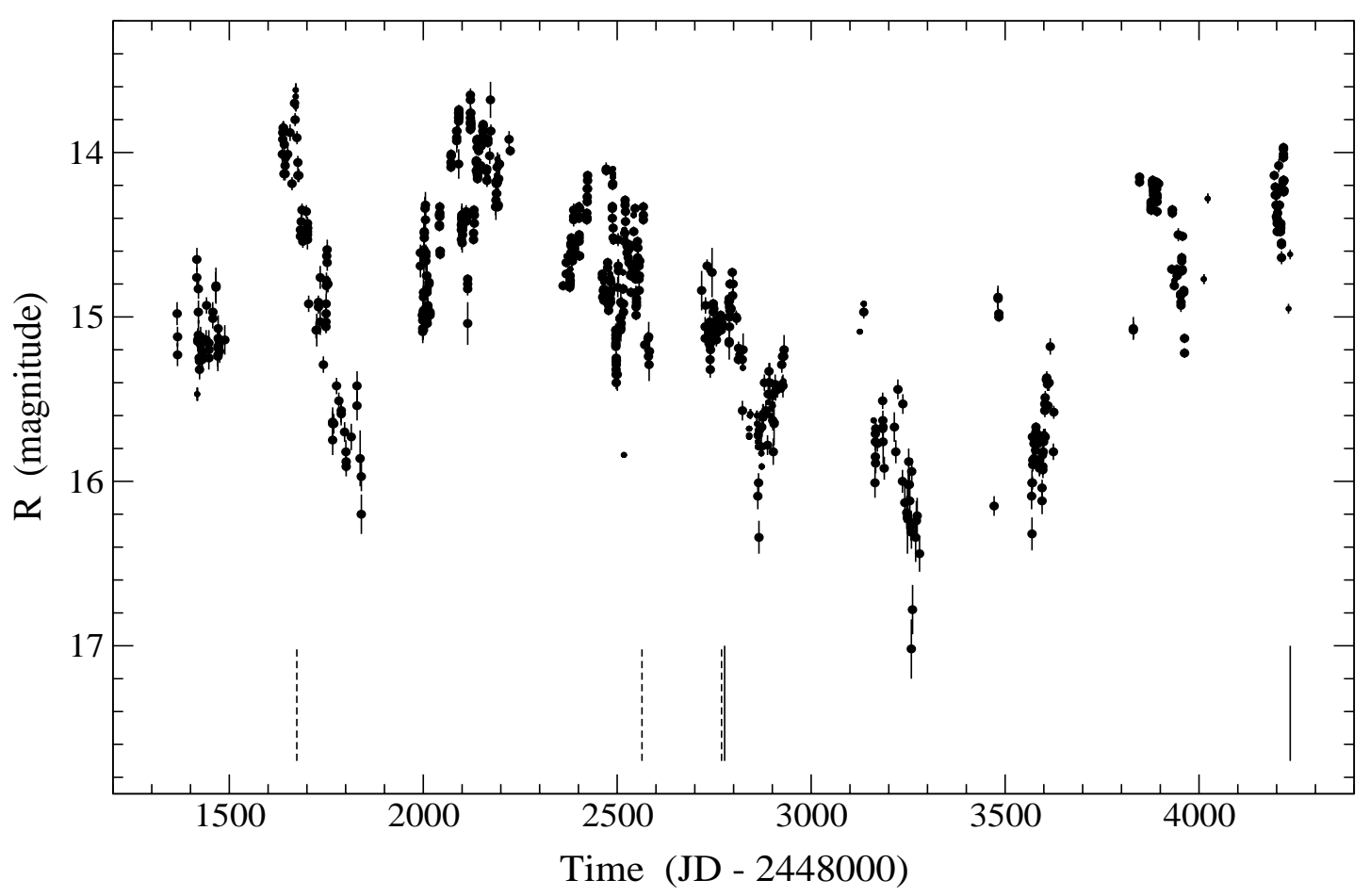

Fig. 1. The light curve of OJ 287 in the $R$ (Cousins) band in the period 1993-2001 from Perugia and Rome databases. The five vertical lines indicate the epochs of the ASCA (1994 Nov. 18, 1997 April 26 and 1997 Nov. 18, dashed lines) and of the two BeppoSAX (1997 Nov. 24,2001 Nov. 21, solid lines) observations.

Later, it was observed by EXOSAT (Giommi et al. 1990) and ROSAT (Urry et al. 1996); the results of these observations have been summarized in a paper by Idesawa et al. (1997), together with the results of an ASCA observation, performed in November 1994 when OJ 287 was in a phase of high optical brightness. Two other ASCA observations were also performed in April and November 1997 (Isobe et al. 2001) during the decline which followed the optical maximum in 1995. BeppoSAX observed OJ 287 twice: in November 1997, as a target of the 1 Jy sample (Padovani et al. 2001), and four years later, in November 2001. These two epochs are indicated in Fig. 1 by two vertical lines. The former pointing was just a few days after the last ASCA observation, while the latter was a TOO triggered by a relatively bright optical state. For both pointings simultaneous optical photometric observations were also performed.

The first X-ray detection of MS 1458+22 (J150101.8+223806) was likely made by Uhuru, because a source at the same position $(4 \mathrm{U} 146+22)$ was reported in the $4 \mathrm{U}$ catalog (Forman et al. 1978), but the presence of other (unresolved) sources in the field of view prevented a reliable flux measurement at that epoch. The source was clearly identified by Stocke et al. (1991) in the Einstein Medium Sensitivity Survey. Although it is a relatively bright BL Lac object, only a few X-ray observations have been performed since the first epoch. On January 22, 1979 Einstein measured the rather low flux (in the $0.5-3.5 \mathrm{keV}$ energy band) of $1.2 \times 10^{-12} \mathrm{erg} \mathrm{cm}^{-2} \mathrm{~s}^{-1}$. The source is present in the RASS (Rosat All Sky Survey) catalogue (1990-91) and it was observed with the ROSAT-PSPC on January 19, 1992 for
$10 \mathrm{ks}$ : in both observations the count rate was very similar $(0.345 \mathrm{c} / \mathrm{s})$ corresponding to a flux of $5 \times 10^{-12} \mathrm{erg} \mathrm{cm}^{-2} \mathrm{~s}^{-1}$ in the 0.1-2.4 keV range (WGACAT, White et al. 1995). BeppoSAX observed MS $1458+22$ in 2001, following a TOO request triggered on the 8th of February when the source reached the magnitude $R=14.62$, the brightest optical state recorded since 1994. In this paper we present the results of the first simultaneous optical and X-ray observation of this BL Lac object, which allow us to study its Spectral Energy Distribution (hereafter SED) over an energy range of about three orders of magnitude.

\section{Observations and data reduction}

\subsection{X-ray observations}

The BeppoSAX observations of OJ 287 were performed on November 25, 1997 and in November 2001. The latter observation started on November 20 (03:19 UT) and ended on November 24 (11:19 UT). The resulting net exposure times were $39.8 \mathrm{ks}$ for the MECS and $23.8 \mathrm{ks}$ for the LECS over a total duration longer than three days, because the efficiency was rather low. The count rate in this pointing was lower than in 1997, but the total statistics were higher because in the first observation the net exposures were $10.7 \mathrm{ks}$ for the MECS and $5.2 \mathrm{ks}$ for the LECS. For the sake of a more accurate comparison we also performed a new spectral analysis of this observation. PDS data were not used because the source fluxes were below the detection limit of the instrument. The observation of MS 1458+22 started on February 19, 2001 (21:55 UT) and 


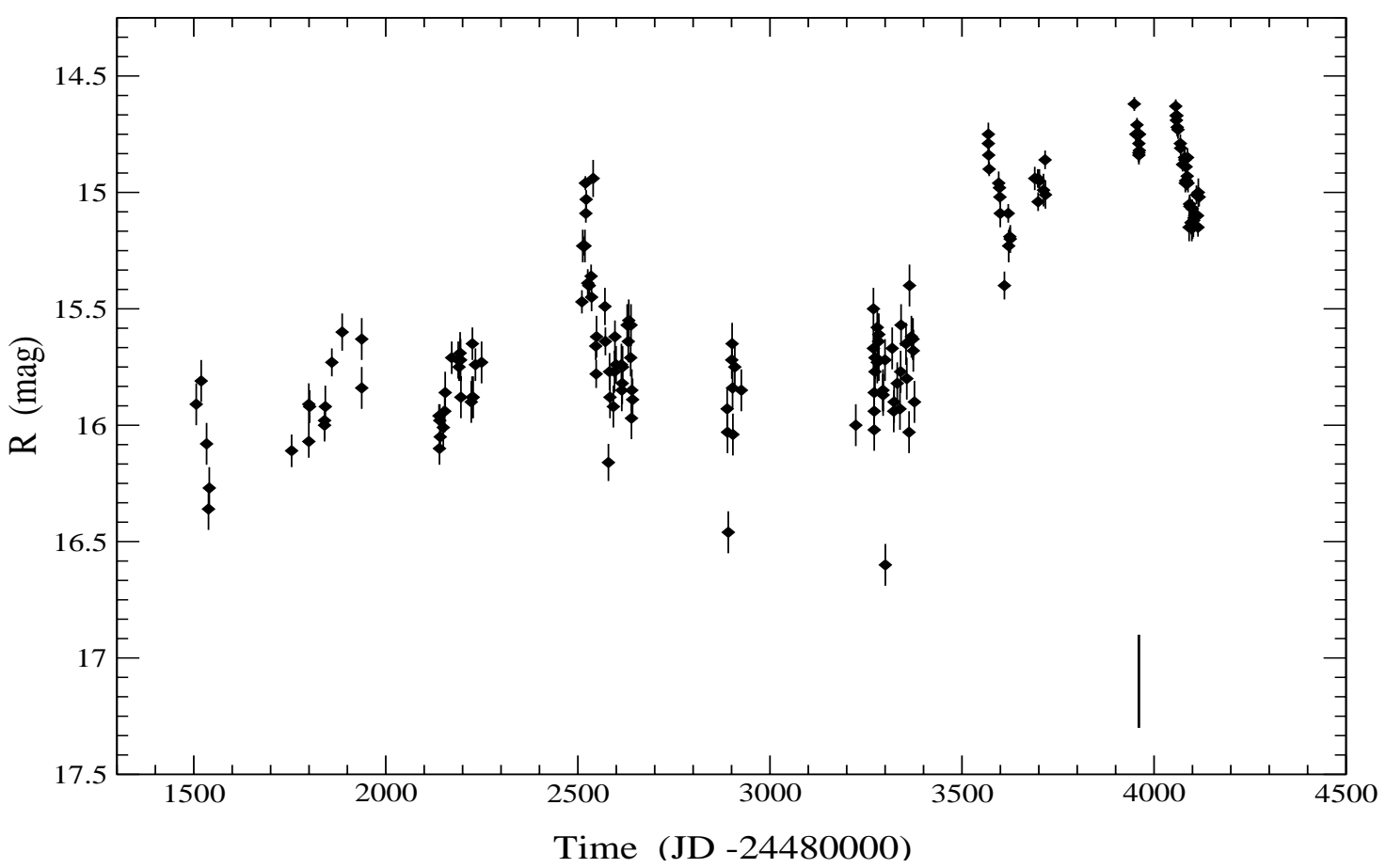

Fig. 2. The light curve of MS $1458+22$ in the $R$ (Cousins) band obtained with the AIT of the Perugia Observatory in the period $1994-2001$. The vertical line indicates the epoch of the BeppoSAX (2001 Feb. 20) observation.

ended on February 21 (01:33 UT): the net exposure for MECS was $47.9 \mathrm{ks}$ and that for the LECS $22.5 \mathrm{ks}$. Also in this case the PDS count rate was too low to detect a signal above the detection limit.

Standard procedures and selection criteria were applied to the data to avoid the South Atlantic Anomaly, solar, bright Earth and particle contamination, using the SAXDAS v. 2.0.0 package. Data analysis was performed using the software available in the XANADU Package (XIMAGE, XRONOS, XSPEC). The images in the LECS and MECS showed a bright pointlike source: events for spectral and timing analysis were selected in circular regions, centred at the source position, with radii of $4^{\prime}$ for both instruments. Background spectra for both instruments were taken from blank fields in the BeppoSAX archive. In all the spectral fits of X-ray data we considered only simple power law models with the low energy absorptions assumed equal to those due to the galactic column densities $N_{\mathrm{H}}=3.04 \times 10^{20} \mathrm{~cm}^{-2}$ for OJ 287 and to $N_{\mathrm{H}}=3.48 \times 10^{20} \mathrm{~cm}^{-2}$ for MS 1458+22, respectively (Dickey \& Lockman 1990).

\subsection{Optical photometry}

The photometric data presented in this paper have been obtained in the course of a monitoring program of a sample of bright blazars carried out since 1994 by our collaboration. The telescopes used were the Automatic Imaging Telescope (AIT, $0.40 \mathrm{~m}$ ) of the Perugia Observatory, the $0.50 \mathrm{~m}$ reflector of the Astronomical Station of Vallinfreda (Rome), the $0.70 \mathrm{~m}$ reflector telescope of the Observatory of Collurania (Teramo), the $0.91 \mathrm{~m}$ Cassegrain telescope of the Astrophysical Observatory of Catania, the $0.70 \mathrm{~cm}$ meniscus telescope of Abastumani Observatory and another reflector $(0.35 \mathrm{~m})$ located near Greve, in Tuscany. The instruments, with the exception of the Catania telescope, are equipped with CCD cameras and standard filters for the Johnson $B, V$ and Cousins $R, I$ bandpasses. The Catania telescope was equipped with a cooled photon-counting photometer and standard Johnson $U, B, V$ filters. Bias, dark, flat field correction and data reduction of CCD frames were performed with IRAF $^{1}$ tasks, and a locally developed software (REDUCE, Fiorucci \& Tosti 1996). Aperture photometry was made with IRAF-apphot or equivalent algorithms and using a sequence of reference stars in the same field of the sources. The $V, R, I$ magnitudes of these stars are given in Fiorucci \& Tosti (1996a) and Fiorucci et al. (1998), while the $B$ values can be found in Gozales-Peres et al. (2001). The Catania photometric data were reduced to the Johnson standard system by using the brightest stars of the CCD sequences and by adding some brighter stars in nearby fields. For all these stars $U, B, V$ magnitudes were improved by means of photometric standard stars from Landolt (1992).

\section{Results}

\subsection{OJ 287}

In November 1997 the unabsorbed X-ray flux of OJ 287 was $F(2-10 \mathrm{keV})=(2.1 \pm 0.3) \times 10^{-12} \mathrm{erg} \mathrm{cm}^{-2} \mathrm{~s}^{-1}$, about $50 \%$ higher than in November 2001 when it was $(1.35 \pm 0.15) \times$ $10^{-12} \mathrm{erg} \mathrm{cm}^{-2} \mathrm{~s}^{-1}$. The spectral analysis of the latter observation was performed using the data in the range $(0.4-1.65) \mathrm{keV}$

${ }^{1}$ IRAF is distributed by the National Optical Astronomy Observatories, which are operated by the Association of Universities for Research in Astronomy, Inc., under cooperative agreement with the National Science Foundation. 
for the LECS and (2-10) $\mathrm{keV}$ for the MECS. The best fit energy index $\left(F(v)=K v^{-\alpha}\right)$ was $\alpha=0.45 \pm 0.08\left(\chi_{\mathrm{r}}^{2}=1.00\right.$, 21 d.o.f.). It was found marginally flatter than in 1997 when it was $0.63 \pm 0.12\left(\chi_{\mathrm{r}}^{2}=0.99,17\right.$ d.o.f.), as shown in the SEDs of Fig. 3, but the uncertainties are too large to safely establish this difference. Again, a similar spectral index, $0.62 \pm 0.05$, was measured by ASCA in November 1994, when the (2-10 keV) flux was about $5 \times 10^{-12} \mathrm{erg} \mathrm{cm}^{-2} \mathrm{~s}^{-1}$ (Idesawa et al. 1997; Kubo et al. 1998), and also in 1997 (Isobe et al. 2001) when the source flux was comparable to that measured by BeppoSAX.

Simultaneous optical photometric measurements were obtained only for the observation of November 2001. Those carried out during the three days of this BeppoSAX pointing showed only very small changes in the optical brightness: the measured magnitudes on November 20 were $I=13.99 \pm 0.01$, $R=14.59 \pm 0.01, V=15.04 \pm 0.03, B=15.61 \pm 0.01$, $U=15.06 \pm 0.07$. A fading of about 0.08 mag was observed on November 22, but the following day the source recovered to the same level as before. The corresponding $v F(v)$ values are also shown in Fig. 3, corrected for the reddening with $A_{V}=0.09$ and using the extinction law by Cardelli et al. (1989). A power law fit to these data gives the energy index $\alpha=$ $1.53 \pm 0.03$. For the BeppoSAX pointing of November 1997 no simultaneous optical data are in our archive. The closest optical photometry was on November 18, a week before the X-ray observation and the subsequent one was on December 5. The former magnitudes were $I=14.38 \pm 0.03, R=15.04 \pm 0.03$, $V=15.53 \pm 0.05$, about $0.5 \mathrm{mag}$ fainter than in 2001. In that period OJ 287 was fading after the second large burst occurred in December 1995-January 1996 and its typical $R$ magnitude was around 15.0 (see Fig. 1). One can reasonably assume that the values given above are representative of the mean state of the source, although large amplitude and fast changes cannot be excluded.

These data indicate that there is no stable correlation between the optical brightness and the X-ray emission when the latter is due to the IC scattering. Model implications will be discussed in the last section.

\section{2. $M S 1458+22$}

A light curve of MS $1458+22$ in the $R$ band covering the period 1994-2001 is shown in Fig. 2; all these data were obtained with the AIT of the Perugia Observatory. It is possible to see that from 1994 to 1998 the typical source magnitude varied between 15.5 and 16.5 , but occasionally it can be even brighter. A first flare was observed in January 2000, when it reached $R=14.75$, after which it remained brighter than 15.5 and on February 8, 2001 it reached the magnitude $R=14.62$, the highest state observed since the beginning of the monitoring, and on this occasion we requested a TOO pointing of BeppoSAX. The X-ray observation was performed after about 10 days, when MS $1458+22$ was still quite bright. On that occasion the source was observed in the optical with the Catania telescope (from about UT $2^{\mathrm{h}}$ to $3^{\mathrm{h}}$ of February 20) and the following magnitudes were measured: $V=15.19 \pm 0.06$, $B=15.53 \pm 0.05$ and $U=14.99 \pm 0.05$ and also with the

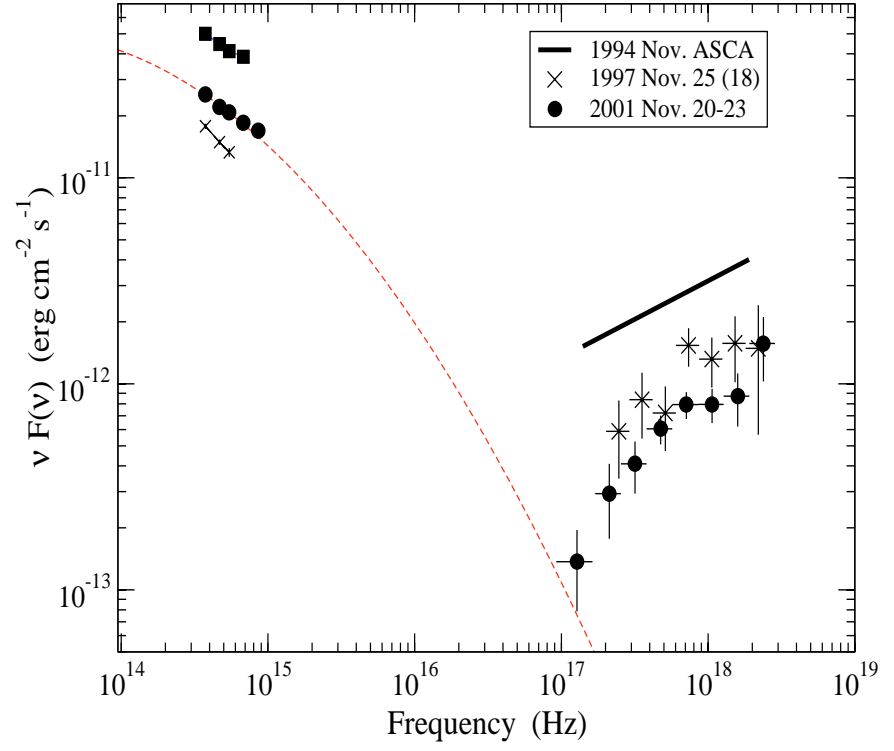

Fig. 3. The X-ray Spectral Energy Distributions of OJ 287 observed in the two BeppoSAX (1997 Nov. 24, 2001 Nov. 21) and ASCA (1994 Nov. 18, Idesawa et al. 1997) pointings. Optical data for all these measurements are also reported using the same symbols, filled squares correspond to the ASCA observation. The optical data of 1997 (the first BeppoSAX observation) were obtained on Nov. 18 and those for the ASCA epoch were taken from Idesawa et al. (1997).

Greve reflector (February 21, $2 \mathrm{~h}$ UT) which gave the magnitudes $I=14.29 \pm 0.03, R=14.79 \pm 0.03$ and $V=15.17 \pm 0.04$.

X-ray data were obtained from LECS $(0.3-1.65 \mathrm{keV})$ and MECS (1.6-9.5 keV) and were found to be well fitted by a single power law with the steep energy index of $1.71 \pm 0.13$ $\left(\chi_{\mathrm{r}}^{2}=1.01,22\right.$ d.o.f.). The corresponding unabsorbed flux in the $2-10 \mathrm{keV}$ band is $(7.5 \pm 0.6) \times 10^{-13} \mathrm{erg} \mathrm{cm}^{-2} \mathrm{~s}^{-1}$. The optical to X-ray SED of MS $1458+22$ is shown in Fig. 4. The above flux corresponds to $3.4 \times 10^{-12} \mathrm{erg} \mathrm{cm}^{-2} \mathrm{~s}^{-1}$ in the ROSAT $(0.1-$ $2.4 \mathrm{keV}$ ) band and to $3.0 \times 10^{-12} \mathrm{erg} \mathrm{cm}^{-2} \mathrm{~s}^{-1}$ in the EINSTEIN $(0.5-3.5 \mathrm{keV})$ band. Therefore we found the source to be fainter than at the epoch of the ROSAT pointing, but brighter than during the EINSTEIN observation. The energy index in the ROSAT observation was $2.0 \pm 0.1$, rather close to our result. The optical spectrum is also fitted by a power law, but the energy spectral slope is $0.99 \pm 0.06$, much flatter than in the X-ray range.

\section{Discussion}

The apparent differences between the SEDs of these two BL Lac objects provide useful information for a better understanding of the origin and dynamics of the non-thermal emission. In both cases the simple power law extrapolations of the optical data into the X-ray range are higher than the measured fluxes. This implies that the spectral index must steepen significantly toward the UV-X range, unless the emission originates in different components. This is, for instance, the case of OJ 287 in which optical photons are due to synchrotron radiation while $\mathrm{X}$-rays are from IC, whereas in MS $1458+22$ both optical and $\mathrm{X}$ radiations belong to the same synchrotron component. 


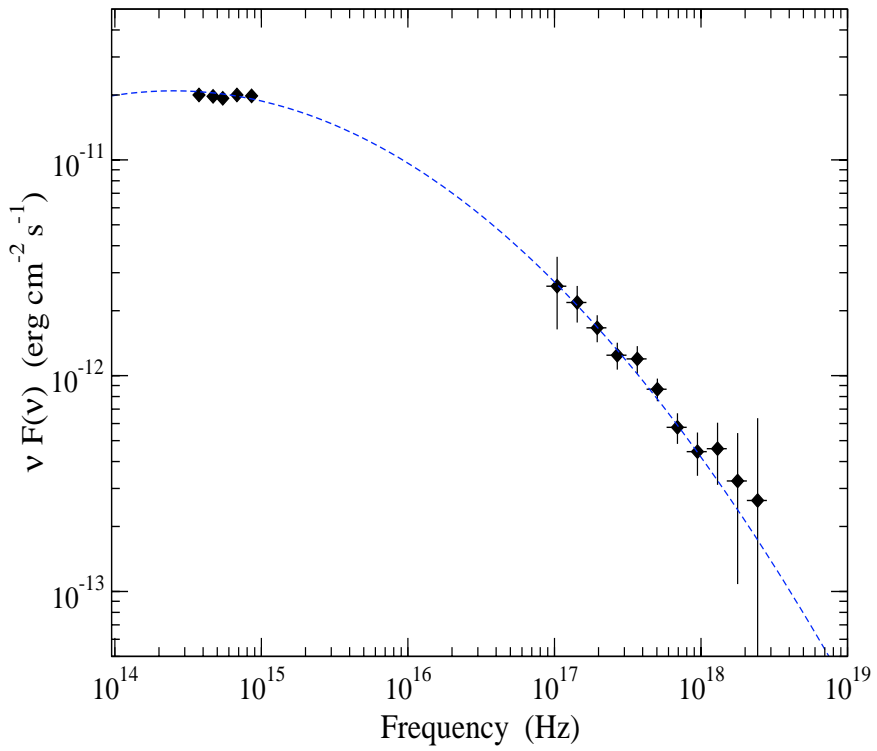

Fig. 4. The X-ray Spectral Energy Distribution of MS 1458+22 observed by BeppoSAX in February 2001, together with simultaneous optical data. The dashed line is a log-parabolic fit of the entire SED.

A simple wide band spectral distribution for the synchrotron component can be obtained using a logarithmic parabola, for which a good parametrisation is:

$\log (v F(v))=\log \left(v_{\mathrm{p}} F\left(v_{\mathrm{p}}\right)\right)-b\left[\log \left(\frac{v}{v_{\mathrm{p}}}\right)\right]^{2}$

where $v_{\mathrm{p}}$ is the peak frequency in the SED and $b$ a curvature parameter.

The use of a log-parabolic spectral distribution for BL Lacs and other Blazar sources was introduced by Landau et al. (1986), who showed that the wide band spectra, from millimetric to optical-UV, of this class of sources are fitted by such a law better than others. More recently Tanihata (2001) confirmed that a log-parabolic distribution gives very good fits for other BL Lac sources. It is possible to show that a logparabolic spectrum is naturally obtained by a statistical acceleration mechanism when the probability for a particle to remain inside the acceleration region is assumed to decrease with a power of the particle energy (Massaro 2002). The parametrisation of Eq. (1), furthermore, is also well suited to describe the spectral changes during the outbursts because the changes of the main source parameters, like energy, number and acceleration rate of the radiating electrons, correspond to changes of the peak frequency $v_{\mathrm{p}}$, of the flux $F_{\mathrm{p}}$ and of the spectral curvature $b$, respectively. A further advantage of this model is that it provides a reasonable estimate of $v_{\mathrm{p}}$, even if it lies outside the observed range.

Our data cover only rather limited frequency intervals and therefore they do not constrain a particular spectral distribution but are compatible with some possible models like a broken power law or other curved laws. In particular, for OJ 287 only the optical points belong to the synchrotron component whereas the measured fluxes in the X-ray range are on the Compton branch and represent only upper limits for the high frequency synchrotron tail. Log-parabolic distributions adapted

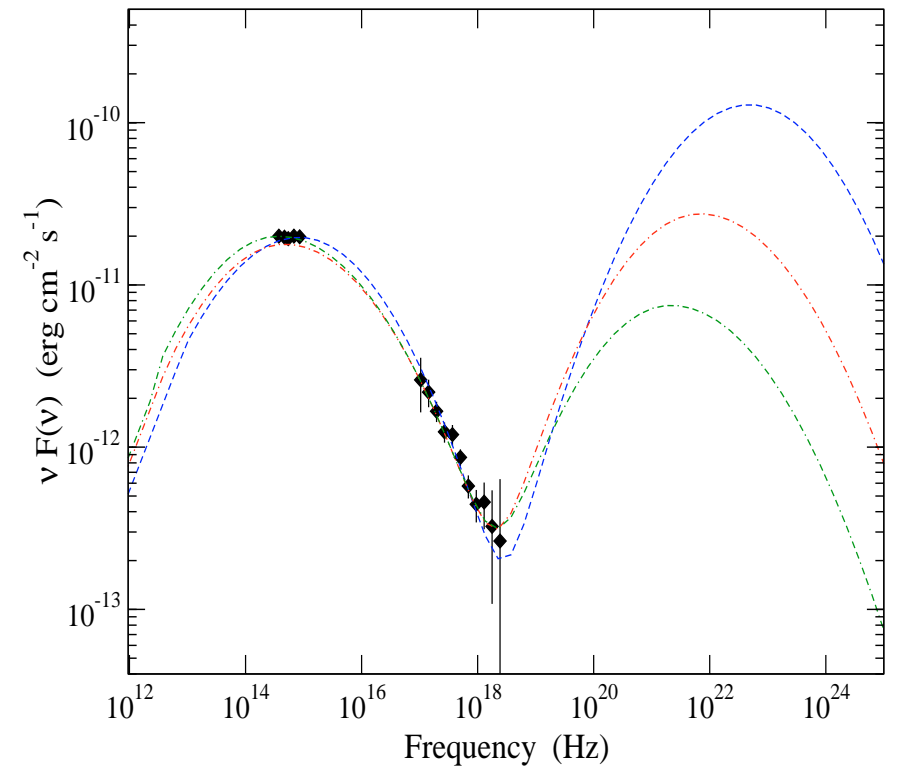

Fig. 5. Possible Spectral Energy Distributions of MS 1458+22 derived using a SSC model. A rather small change of the model parameters, all providing a good fit of the optical and X-ray data, corresponds to very different fluxes and peak frequencies of the Compton peak.

to the optical to X-ray SEDs of OJ 287 and MS 1458+22 are shown in Figs. 3 and 4, respectively: in the case of the former source the curve has been chosen taking into account that the synchrotron component is not detected in the X-ray range, while for the latter it fits all the data. The most relevant difference is in the estimated position of the peak frequency which lies at about $2.2 \times 10^{13} \mathrm{~Hz}(14 \mu \mathrm{m})$ for OJ 287 and at $2.5 \times 10^{14} \mathrm{~Hz}(1.2 \mu \mathrm{m}$, approximately in the $J$ band $)$ for MS 1458+22. Also different, but not so large, are the values of the curvature parameter $b$ equal to 0.13 for MS $1458+22$ and larger than 0.20 for OJ 287, respectively. We stress that these values agree well with those that can be derived from the fit parameters of the LBL sample considered by Landau et al. (1986), for which the $b$ values range from 0.09 to 0.22 with the mean value of 0.14 . This finding suggests that the SEDs of the synchrotron component, at least in LBL sources, have remarkably similar shapes, likely because the same acceleration mechanism is active. Similar values of the curvature parameter are also found for the HBL (High energy peaked BL Lac) sources observed with BeppoSAX in bright states, whereas, when not in outburst, they frequently have $b$ values larger than $\sim 0.3$ (Giommi et al. 2002). The smallest curvature for HBL sources was found for Mkn 501 where it varied in the range 0.1-0.3, like a LBL object (Massaro et al., in preparation).

A comparison between the two BeppoSAX observations (crosses and filled circles in Fig. 3) indicates that the flux variation in the optical could be opposite to that observed in the $\mathrm{X}$-rays: precisely, the source was more luminous in the optical when it was fainter in the X-ray band. Unless OJ 287 in November 1997 underwent a brightness increase, not observed because of the sampling, like that of November 2001, one could infer that there is no direct positive correlation between the optical synchrotron emission and the IC X-rays. It is interesting 
to compare these results with those of a previous ASCA observation, performed on November 18, 1994 (Idesawa et al. 1997; Kubo et al. 1998). On that occasion OJ 287 was even brighter than in 1997, both in the optical and in the X-ray range, but with a similar spectral index. In an early observation performed with EXOS AT in February 1984 (Sambruna et al. 1994), the source showed a quite high X-ray flux and a photon index around 2.3, much steeper than in our observations. From the historic light curve (Pursimo et al. 2000) we can see that in the beginning of 1984 OJ 287 was very bright, being in the secondary burst phase. It is then likely that the synchrotron component was bright enough to be dominant at energies of a few $\mathrm{keV}$.

For the case of MS 1458+22, where the synchrotron component is better described by our data, we tried to evaluate the expected $\gamma$-ray SED in the framework of a homogeneous Synchro-Self-Compton (SSC) emission from a jet. The model involves a population of relativistic electrons emitting synchrotron radiation in a single zone of a jet that is moving at relativistic speed and at a small angle to the line of sight. These photons are scattered at higher energies via IC process by the same electron population. The physical parameters that define the model are the jet radius, the Doppler factor $\delta$, the magnetic field $B$ and the density and the spectral parameters of the electron population. The Klein-Nishina cross section is used in the computation of the Compton scattering. A detailed description of this SSC model can be found in Perri (2002).

This model contains some parameters which cannot be constrained by the knowledge of the synchrotron component only, therefore to compute the SED of MS 1458+22 we fixed some of them to acceptable physical models: so we take a jet radius of $10^{-3} \mathrm{pc}$ and a beaming factor of 20 , while the magnetic field $B$, the density $n$ and the upper cutoff Lorentz factor $\gamma_{\mathrm{b}}$ of the electrons spectrum (about equal to that of the electrons radiating at the SED peak frequencies) were left free. Three possible resulting SEDs, all giving an acceptable fit of the low energy component, are shown in Fig. 5: one corresponds to $B=0.32 \mathrm{G}, n=4.5 \times 10^{2}$ electrons $\mathrm{cm}^{-3}$ and $\gamma_{b}=6.6 \times 10^{3}$ (dashed/blue line), another to $B=0.8 \mathrm{G}, n=2.5 \times 10^{2}$ electrons $\mathrm{cm}^{-3}$ and $\gamma_{b}=3.3 \times 10^{3}$ (dashed-dotted/red line), and the third one to $B=1.4 \mathrm{G}, n=2.0 \times 10^{2}$ electrons $\mathrm{cm}^{-3}$ and $\gamma_{b}=2.1 \times 10^{3}$ (double dashed-dotted/green line). The resulting Compton spectra differ largely both for the intensity and the peak frequency and do not allow a satisfactory prediction of the $\gamma$-ray luminosity, because it could vary by more than one order of magnitude. In the case of an HBL source the synchrotron peak would be around $10^{17}-10^{18} \mathrm{~Hz}$ and the Compton peak moves up to the TeV range.

Simultaneous observations over the entire electromagnetic spectrum represent the best and likely only way to obtain a more complete estimate of the model parameters. In the coming years, when the planned space missions for $\gamma$-ray astronomy, like GLAST and AGILE, will be in operation, a large international effort should be done to organize a stable multifrequency monitoring of a sample of these sources. Finally, we stress that it is useful to observe sources like OJ 287 not only in bright states but, in particular, when they are faint. Only in this case could the IC component be dominant in the X-ray range and one will have the possibility to study if it is correlated with another spectral component, necessary for understanding which electron and photon populations are responsible for this radiation.

Acknowledgements. The authors are grateful to the referee, Dr. K. Ghosh, for his critical review of the manuscript. Part of this work was performed with the financial support Italian MIUR (Ministero dell' Istruzione Universitá e Ricerca) under the grant Cofin 2001/028773.

\section{References}

Cardelli, J. A., Clayton, G. C., \& Mathis, J. S. 1989, ApJ, 345, 245

Dickey, J. M., \& Lockman, F. J. 1990, ARA\&A, 28, 215

Fiorucci, M., \& Tosti, G. 1996, PUO Int. Rep. N., 6

Fiorucci, M., \& Tosti, G. 1996a, A\&AS, 116, 403

Fiorucci, M., Tosti, G., \& Rizzi, N. 1998, PASP, 110, 105

Forman, W., Jones, C., Cominsky, L., et al. 1978, ApJS, 38, 357

Giommi, P., Barr, P., Garilli, B., et al. 1990, ApJ, 356, 432

Giommi, P., Massaro, E., Chiappetti, L., et al. 1999, A\&A, 351, 59

Giommi, P., Capalbi, M., Fiocchi, M., et al. 2002, in Blazar Astrophysics with BeppoSAX and Other Observatories, ed. P. Giommi, E. Massaro, \& G. G. C. Palumbo (ASI special publication), 63

Gonzales-Peres, J. N., Kidger, M. R., \& Martin-Luis, F. 2001, AJ, 122, 2055

Idesawa, E., Tashiro, M., Makishima, K., et al. 1997, PASJ, 49, 631

Isobe, E., Tashiro, M., Sugiho, M., et al. 2001, PASJ, 53, 79

Kubo, H., Takahashi, T., Madejski, G., et al. 1998, ApJ, 504, 693

Landau, R., Golish, B., Jones, T. J., et al. 1986, ApJ, 308, 78

Landolt, A. U. 1992, AJ, 104, 340

Massaro, E. 2002, Proc. Blazar Astrophysics with BeppoSAX and Other Observatories, ed. P. Giommi, E. Massaro, \& G. C. C. Palumbo (ASI special publication), 3

Padovani, P., \& Giommi, P. 1995, ApJ, 444, 567

Perri, M. 2002, Ph.D. Thesis, University La Sapienza, Roma

Pursimo, T., Takalo, L. O., Sillanpää, A., et al. 2000, A\&AS, 146, 141

Sambruna, R. M., Barr, P., Giommi, P., et al. 1994, ApJS, 95, 371

Sillanpää, A., Haarala, S., Valtonen, M. J., et al. 1988, ApJ, 325, 635

Stickel, M., Fried, J. W., \& Kühr, H. 1989, A\&AS, 80, 103

Stocke, J. T., Morris, S. L., Gioia, I. M., et al. 1991, ApJS, 76, 813

Tagliaferri, G., Ghisellini, G., Giommi, P., et al. 2000, A\&A, 354, 431

Tanihata, C. 2001, Ph.D. Thesis, ISAS Res. Note, 739

Urry, M., Sambruna, R. M., Worral, D. M., et al. 1996, ApJ, 463, 424

Valtaoja, E., Teräsranta, H., Tornikoski, M., et al. 2000, ApJ, 531, 744

White, N. E., Giommi, P., \& Angelini, L. 1995, BAAS, 26, 1372 